\title{
Moisture Induced Antiplasticization of Microcrystalline Cellulose
}

S.P. Chamarthy, T. Carvajal and R Pinal

Purdue University, Industrial and Physical Pharmacy, 575 Stadium Mall Dr.

West Lafayette, IN 47907, USA.

carvajal@pharmacy.purdue.edu

\begin{abstract}
The antiplasticizing effect of water on the permeability, crystallinity and mechanical properties of microcrystalline cellulose (MCC) was investigated. Moisture decreases the permeability of water and octane up to a critical relative humidity (RH) of 5\% and increases tensile strength and crystallinity up to $33 \% \mathrm{RH}$. The results are clear indications of the antiplasticization of MCC by water and are consistent with the quasicrystallinity antiplasticization model. The different critical RH values can be attributed to the scale of scrutiny of the measured property. Moisture induced antiplasticization has important implications where dry conditions are desired. A carefully chosen (low) water content, being both easier to achieve, and more economical, could be as effective or even better than complete dryness.
\end{abstract}

Keywords: Antiplasticization, MCC, Water, Permeability, Mechanical properties

Introduction: Microcrystalline cellulose (MCC) is one of the most commonly used carbohydrate based polymers in pharmaceutical tablet manufacturing. Exposure of MCC to moisture, whether as part of the manufacturing process or due to environmental exposure during handling and storage, is expected to alter the mechanical performance of MCC. It has been established that at low concentrations, plasticizers often act as antiplasticizers (Sears 1982; Seow 1999), and virtually no investigations on this phenomenon have been conducted in the pharmaceutical field. In this study, we investigate the effect of low concentrations of water on MCC. The study covers the antiplasticizing effect of water on MCC at both the microscopic and macroscopic levels. For investigating the phenomenon at the molecular level, bulk diffusion of two very different vapor probes, water and octane, was used. For the study of antiplasticization at the macroscopic level, the mechanical properties (tensile strength and Young's modulus) of MCC as functions of moisture content were used.

Results and discussion: The bulk diffusion constant (permeability, $D_{p}$ ) of water in MCC, as a function of relative humidity $(\mathrm{RH})$, was obtained from the kinetics of water sorption observed at each RH. The results are shown in Figure 1. The permeability of water initially decreases with water content until $\sim 5 \% \quad \mathrm{RH}$, followed by an increase under higher $\mathrm{RH}$ conditions. A decrease in water permeability indicates that low amounts of water actually increase the rigidity of the polymer matrix. At first, water impedes its own transport through the polymer, before higher moisture levels begin to facilitate permeation.

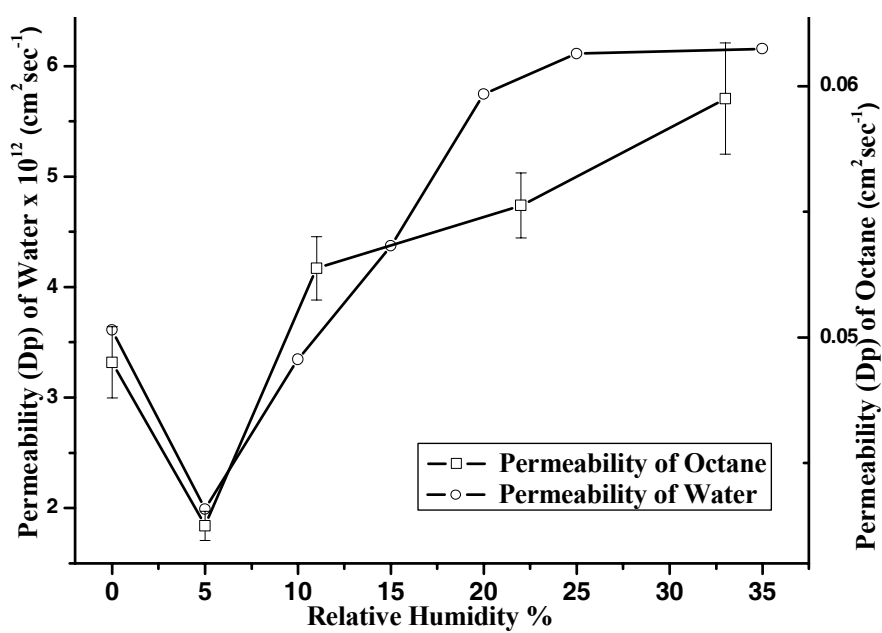

Figure 1. Permeability of water (from moisture sorption isotherm) and octane (by IGC) in MCC as function of RH.

independent confirmation of the same phenomenon is also shown in Figure 1. The permeability of octane, a highly hydrophobic molecule is also shown. In this case, permeability was determined using inverse gas chromatography (IGC)(Van Deemter 1956; Thielmann 1999). From the figure, it is clear that at low concentrations (up to 5\% RH), water impedes the permeation of octane, while higher moisture contents facilitate it. The fact that the same result was obtained using two very different probes (water and octane), using completely different instrumental techniques, and data treatment, provides independent confirmation of antiplasticization at the molecular level. 
The triple point beam bending method was used to measure the tensile strength and Young's modulus of compacts made from MCC samples pre-equilibrated under different RH conditions. The compacts were made under identical conditions, such that the only difference among them was their water content. The results plotted in Figure 2 clearly show antiplasticization, i.e., at low concentrations, water increases tensile strength and Young's modulus up to a turnabout value, beyond which water acts as a plasticizer. Figure 3 also shows the crystallinity index of MCC determined by FT-IR(Ek 1995). Crystallinity of MCC increases with water content reaching a plateau. The onset of the plateau coincides with the turnabout value for tensile strength. These results suggest that mechanical antiplasticization of MCC occurs for as long as the crystallinity of the polymer increases upon addition of the plasticizer.

\section{CONCLUSIONS:}

The antiplasticizing effect of water is clearly observable on the macroscopic (mechanical) and microscopic (permeability) properties of MCC as an increase in mechanical strength and a decrease in permeability, respectively. Our observations are consistent with the quasi-crystallinity antiplasticization model (Ghersa 1958; Guerrero 1989), which suggests that an increase in local order (crystallinity) with the addition of a plasticizer is responsible for the antiplasticizing effect. It is noteworthy that the



Figure 2. Tensile strength, Young's modulus and crystallinity of MCC as a function of RH. turnabout values for antiplasticization are different for different properties. A plausible explanation is that the threshold value will depend on the scale of scrutiny reflected by the measured property. A microscopic property, which measures the behavior of molecules, will have a low threshold. Conversely, a property like tensile strength, which reflects the cohesive energy of the entire matrix, will have a higher threshold. The obtained results are significant in systems where low moisture levels are required. A carefully selected water content, while easier to achieve and more economical, could be as effective or even better than complete dryness.

\section{Acknowledgment:}

NSF I/UCRC Award No. 0003064.

\section{Reference List}

Ek, R., P. Wormald, J. Ostelius, T. Iversen and C. Nystrom (1995). "Crystallinity Index Of Microcrystalline Cellulose Particles Compressed Into Tablets." Int. J. Pharm. 125(2): 257-264.

Ghersa, P. (1958). "Effect of small quantities of plasticizers in PVC compounds." Mod. Plast. 36: $135-142$.

Guerrero, S. J. (1989). "Antiplasticization and Crystallinity in Polyvinyl-Chloride)." Macromolecules 22(8): 3480-3485.

Sears, J. K. and J. R. Darby (1982). The Technology of Plasticizers. New York, John Wiley \& Sons.

Seow, C. C., P. B. Cheah and Y. P. Chang (1999). "Antiplasticization by water in reducedmoisture food systems." J. Food Sci. 64(4): 576-581.

Thielmann, F. and V. McClean (1999). Determination of permeability coefficients of alkanes in polyethylene powder by infinite dilution inverse gas chromatography. IGC Application Note 206. London, Surface Measurement Systems.

Van Deemter, J. J., F. J. Zuiderweg and A. Klinkenberg (1956). "Longitudinal diffusion and resistance to mass transfer as causes of nonideality in chromatography." Chem. Eng. Sci. 5: 271-289. 